\title{
An improved method of Retinex for night color image enhancement
}

\author{
Ying Tao ${ }^{1}$, Jin Duan ${ }^{2, a}$, Xiaowei Liang ${ }^{2}$,Yinghui Sun ${ }^{2}$,Xiaoting Gong ${ }^{2}$ \\ ${ }^{1}$ School of Electronic and Information Engineering, Changchun University of Science and \\ Technology, Changchun, JiLin 130022, China \\ ${ }^{2}$ School of Computer Science and Technology, Changchun University of Science and Technology, \\ Changchun, JiLin 130022, China
}

Corresponding author: duanjin@vip.sina.com

Keywords: Image enhancement,Adaptive brightness adjustment,Tri-gaussian model.

\begin{abstract}
Images collected at night are usually characterized by low contrast, low brightness, missing numerous details and so on. In particular, an improved method of multi- scale Retinex is proposed for the case where there is a local light source, a large number of dark areas and high luminance regions in the image. Firstly, the adaptive brightness adjustment is adopted to replace the logarithmic transformation to compress dynamic range of the brightness components. Then, tri-gaussian model of the retinal neuron is introduced into the estimation of illumination image, and a bilateral filtering Retinex algorithm based on the tri-gaussian model and traditional Gauss filter is proposed. The image's edge detail can be better preserved while the contrast is effectively enhanced. At the same time, the color of the enhanced image can be maintained. Experimental results show that the algorithm is superior to other algorithms.
\end{abstract}

\section{Introduction}

In 1964, Edwin.H.Land, an American physicist, first proposed the Retinex ${ }^{[1]}$ theory, also known as color constancy theory. It is used to describe the color perception model of the human visual system, and it also provides an effective theoretical basis for image dynamic range enhancement, color retention and details of information recovery. Land believes that the colour of the human visual system is only related to the reflection on the surface of object, not the intensity of incident light. Therefore, it is the core process of Retinex algorithm to extract the incident light part from the original image and then deal with the reflected component of the scene. According to the difference of methods for estimating the Incident component, the existing Retinex algorithms mainly include: random path Retinex, central/surrounding Retinex, MsCann's Retinex, single/multi-scale ${ }^{[2,3]}$ Retinex, and Retinex algorithm with color recovery.

This paper presents an improved method of multi-scale Retinex. The flow chart of this algorithm is shown in Fig. 1. Night images contain a large number of connected dark and high brightness areas, this paper proposes an adaptive brightness adjustment function of dynamic range compression, so that images can be non-linear adjustment according to the brightness of the image. In addition, we propose a Retinex algorithm for bilateral filter of tri-gaussian filter and Gauss filte, which preserves the advantages of bilateral filter and improves the edge processing effect of image enhancement. 


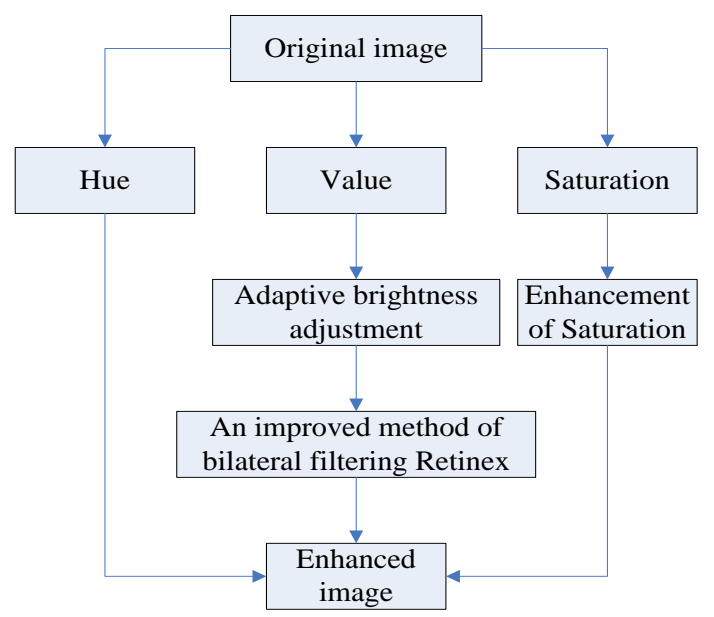

Fig. 1 Flowchart of the algorithm in this paper

\section{Adaptive brightness adjustment}

Adaptive brightness adjustment is mainly based on the brightness of the original image, and it is used to achieve dynamic range compression by nonlinear mapping. In the classical MSR, the dynamic range compression of the image is realized by using the logarithm function. However, according to the characteristics of the logarithmic function, the dynamic range of the dark area in the image is stretched, and the dynamic range of the highlight area is compressed, so that details of the dark area of the image are obviously enhanced. In this paper, an adaptive brightness adjustment function is proposed, which divides the dark area and the bright region by a fixed threshold. Different brightness adjustment functions are used for the two regions, so that the dark area and the bright area are stretched, while only the middle is compressed. The function is as follows:

$$
\begin{gathered}
m \log [I(x, y)]=\left\{\begin{array}{c}
w_{L} \cdot \log [I(x, y)+1], I(x, y) \leq T h \\
-w_{H} \cdot \log [D-I(x, y)]+\log D, I(x, y)>T h
\end{array}\right. \\
w_{L}=\frac{\frac{T h}{D-1} \cdot \log D}{\log (T h+1)}, w_{L}=\frac{\left(1-\frac{T h}{D-1}\right) \cdot \log D}{\log (D-T h)}
\end{gathered}
$$

Among them, $\mathrm{D}$ is the grayscale dynamic range of the image. $\omega_{L}$ and $\omega_{H}$ are the weights of the dark and bright regions respectively. Th is the brightness segmentation threshold, and when the equation Th $=\mathrm{D}-1$ is satisfied, the function degenerates into a normal logarithmic function.

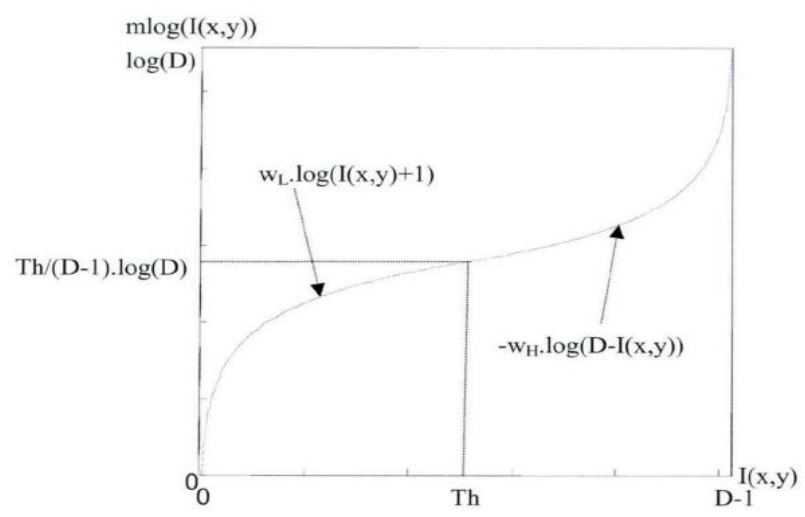

Fig. 2 Characteristics of dynamic range compression

Fig. 2 shows the characteristic curve of the adaptive brightness adjustment function. As you can see from the diagram, the pixel brightness value of the dark area is less than the threshold Th, the dynamic range is stretched, so the contrast in the dark area becomes larger. The bright area is the same. That is, it can achieves the details of the dark area and highlight by the non-linear adjustment. 


\section{Bilateral filtering Retinex based on tri-gaussian model and Gauss filter}

\subsection{Tri-gaussian model of the retinal neuron}

In order to quantitatively describe the input-output characteristics of retinal ganglion cells, Rodieck $^{[4]}$ proposed a double-Gaussian difference model (DOG model), with concentric overlap, different sizes of circular area to describe the traditional ganglion cells in the central area and peripheral area. However, in the traditional sense field, there is a large range of disinhibition regions, and $\mathrm{Li}^{[5]}$ proposed the tri-gaussian model on the basis of DOG model. The third gaussian kernel is used to represent large scale inhibitory regions. The tri-gaussian model is as follows:

$$
G_{r}(x, y)=\alpha_{1} \exp \left(-\frac{x^{2}+y^{2}}{2 \delta_{1}^{2}}\right)-\alpha_{2} \exp \left(-\frac{x^{2}+y^{2}}{2 \delta_{2}^{2}}\right)+\alpha_{3} \exp \left(-\frac{x^{2}+y^{2}}{2 \delta_{3}^{2}}\right)
$$

Among them, $\alpha_{1}, \alpha_{2}, \alpha_{3}$ respectively represent the peak coefficients of the central, peripheral and peripheral. $\delta_{1}, \delta_{2}, \delta_{3}$ respectively represent the scale parameters of the center, surrounding and periphery. This model can be used to deal with the brightness contrast edge, which can not only enhance the edge contrast, but also effectively enhance the brightness contrast and brightness gradient information which is filtered by the peripheral mechanism of the traditional receptive field center.

The characteristics of tri-gaussian model in its spatial domain are shown in Fig. 3, which reflect the distribution of sensory characteristics of the central excitatory, peripheral inhibitory and peripheral excitatory retinal ganglia.


Fig. 3 Spatial structure of tri-gaussian model

\subsection{An improved method of bilateral filtering Retinex}

In order to better estimate the illumination component, the paper adopts the Retinex method based on the tri-gaussian model and the traditional gaussian filter. This kind of bilateral filtering not only considers the similarity between the neighborhood pixel and the current point in the numerical and position, but also the weight of the neighborhood pixel in combination with the receptive characteristics of the retinal ganglion cells. This makes it possible to better estimate the edge contrast of the illuminance component, to enhance the region illumination contrast and gradient information, so that the estimated illuminance component is more accurate.

For the color image I, the luminance component $\mathrm{S}$ is extracted using the HSV color model:

$$
S=\left(I_{R}+I_{G}+I_{B}\right) / 3
$$

Among them, $I_{R}, I_{G}$ and $I_{B}$ respectively represent red components, green components and blue components of color images.

Obtain the current pixel average illumination value by bilateral filtering:

$$
L(x, y)=\sum_{i, j=-M}^{M} G_{r} G_{v} S\left(x_{i}, y_{i}\right) / \sum_{i, j=-M}^{M} G_{r} G_{v}
$$

Among them, $G_{r}$ is the adjacent tri-gaussian kernel function of space, $G_{v}$ is the traditional gaussian kernel function, and its expression is as follows:

$$
G_{v}(x, y)=\exp \left\{-\frac{\left[S(x, y)-S\left(x_{i}, y_{i}\right)\right]^{2}}{2 \delta_{v}^{2}}\right\}
$$

Among them, $\delta_{v}$ is the scale parameter of numerical gaussian filter, which is used to control the attenuation rate of each weight coefficient. 


\section{Experimental results and analysis}

In order to verify the enhancement effect, the algorithm is compared with four kind of surround Retinex methods, and the result is shown in Fig. 4. In the figure, Figure (a) is the original image, the night color image with the local light source is selected; Figure (b) adopts the single-scale Retinex algorithm. Although the brightness and contrast of the image are improved, there is a significant "halo" situation, and the enhanced image color distortion is serious; Figure (c) usees multi-scale Retinex algorithm, comparing with the SSR algorithm, the dynamic range compression can be done well, but there are still obvious "halo" and color distortion; Figure (d) usese Retinex algorithm with color restoration algorithm, the "halo" phenomenon was restrained, but the overall image showing "white" phenomenon; Figure (e) is bilateral filtering Retinex algorithm ${ }^{[6]}$, solving the "white" phenomenon, the enhanced recovery and the brightness of the whole image color contrast is achieved good results, but a part of the figure tree of fuzzy phenomenon will lead to the loss of the image edge details; Figure (f) adopts the algorithm of this paper, which enhances the brightness contrast of the image, while maintaining the image edge details and the color characteristics of the object itself.

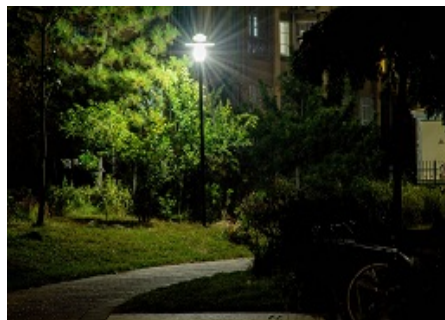

(a) Original image

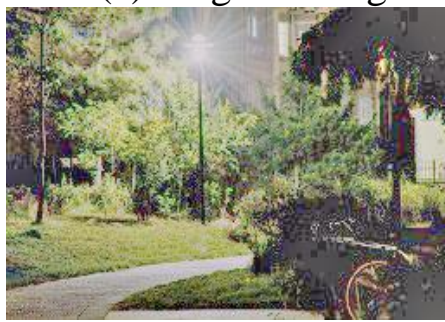

(d) MSRCR

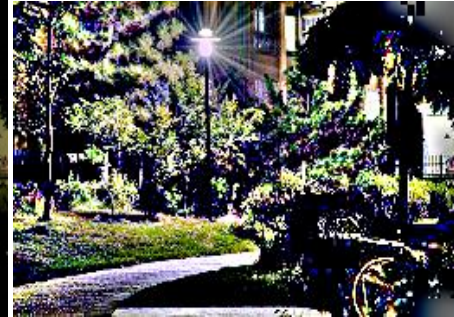

(b) SSR

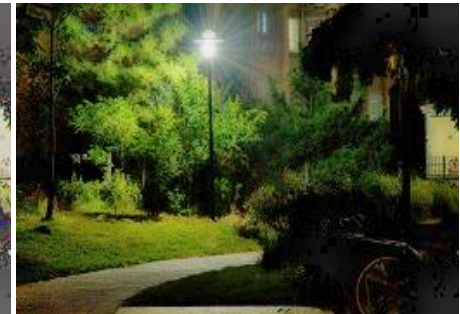

(e) Bilateral filtering Retinex



(c) MSR



(f) The algorithm

Fig. 4 Comparison of image enhancement effects

\section{Conclusion}

This paper presents an improved multi-scale Retinex algorithm, aiming at night color image, especially the night image with local light source. According to the intensity of the image, an adaptive brightness adjustment function is proposed, so that the dark and bright areas of the image are obviously enhanced. This paper proposes a bilateral filter Retinex based on tri-gaussian and gauss model, enhances the image brightness, contrast and edge preserving image details, optimize the existing defects of the traditional bilateral filtering Retinex. The image color is also better restored, which makes the surveillance image easier to identify, is more conducive to criminal detection, and also lays the foundation for subsequent image processing work.

\section{References}

[1] Land E,McCann J.Lightness and retinex theory[J].Journal of the Optical Society of America,1971,61(1):1-11.

[2] Liu Jia-ming,Zhao Yu-ming,Hu Fu-qiao.A nonlinear image enhancement algorithm based on scale Retinex [J].Journal of Shanghai Jiaoton University,2007,41(5):685-688.

[3] Raham Z,Jobson D J,Wodell G A.Multi-scale Retinex for color image enhancement [C].Proceedings of IEEE International Conference on Image Processing,1996,3:1003-1006. 
[4] Rodieck R W.Quantitative analysis of cat retinal ganglion cell response to visual stimuli [J].Vision Reaearch,1965,5(11):583-601.

[5] Li C Y,Pei X,Zhow Y X,et al.Role of the extensive area outside the X-cell receptive filed in brightness information transmission [J].Vision Research,1991,31(9):1529-1540.

[6] Hu W W,Wang R G,Fang S,et al.Retinex algorithm for image enhancement based on bilateral filtering [J].Journal of Engineering Graphics,2010,31(2):104-109(Ch). 